\title{
Diminuição do Estigma sobre Transtorno Mental após Internato em Psiquiatria do Curso de Medicina de Duas Instituições em Fortaleza (CE)
}

\author{
Diminishing the Stigma around Mental \\ Disorder following Medical Internship in \\ Psychiatry at Two Institutions in Fortaleza \\ (CE)
}

\section{PALAVRAS-CHAVE}

- Estigma

- Internato Médico.

- Transtorno Mental.

- Educação Médica.

\author{
Lorena Pinho Feijót \\ Sarah Gadelha Mottal \\ Rui Pimenta Saldanhal \\ Marcos Kubrusly \\ Kristopherson Lustosa Augusto ${ }^{I I}$
}

\begin{abstract}
RESUMO
Historicamente, várias doenças são causadoras de estigma. O estigma e o preconceito em torno da doença mental existem amplamente em todo o mundo e pesquisas têm mostrado que a população em geral tem conhecimentos limitados sobre doenças mentais. Entre os transtornos mentais, a esquizofrenia é uma das mais estigmatizadas. Os estudantes de Medicina, por também fazerem parte da sociedade, não ficam imunes ao estigma em relação a pessoas com transtornos mentais. Vários estudos sugeriram que a educação psiquiátrica do estudante de Medicina, principalmente as experiências que envolvem contato direto com o paciente, como o internato, pode ter um impacto positivo, como no engajamento direto ao atendimento do paciente, interesse em participar de outras atividades como terapia grupal, gerenciamento de casos, além do entendimento de que pacientes com condições psiquiátricas podem ser tratados com sucesso. A maioria dos médicos, no entanto, recebe pouco treinamento ao interagir com pacientes com doenças mentais. Geralmente sentem-se desconfortáveis ou ineficazes ao se comunicarem com eles, mesmo sobre queixas físicas. O objetivo deste estudo foi avaliar se o estágio do internato em um hospital psiquiátrico de Fortaleza diminui o estigma dos alunos de Medicina em relação à doença mental. Foi aplicado o questionário validado AQ-9 aos estudantes de Medicina no período inicial do estágio do internato em Psiquiatria e repetida a mesma avaliação no final do período. Do total de 88 estudantes, observou-se que 37 (42\%) eram do sexo masculino e 51 (58\%) eram do sexo feminino. A média de idade foi de 24,68 anos. Pôde-se observar que houve diferença entre os três primeiros dominios do AQ-9, que evidenciaram, respectivamente, uma diminuição significativa em piedade $(p=0,029)$, periculosidade $(p=0,004)$ e medo $(p<0,001)$. Admite-se que existe estigma na população de estudantes analisada e que o estágio em Psiquiatria do internato de duas faculdades de Medicina estudadas reduziu significantemente três dos nove domínios avaliados. Apenas o gênero como dado sociodemográfico influenciou o resultado. Alunos do sexo feminino apresentaram maior média do que os alunos do sexo masculino em relação ao domínio medo, enquanto os alunos do sexo masculino apresentaram maior média do que os alunos do sexo feminino em relação ao domínio segregação. Fortalece-se a importância do estágio em Psiquiatria durante o internato para além do aprendizado técnico, já que o mesmo tem a capacidade de diminuir o estigma em relação aos pacientes psiquiátricos, principalmente os pacientes esquizofrênicos.
\end{abstract}

Centro Universitário Unichristus, Fortaleza, Ceará, Brasil.

${ }^{I I}$ Centro Universitário Unichristus, Fortaleza, Ceará, Brasil.

Universidade de Fortaleza, Fortaleza, Ceará, Brasil.

Universidade Federal do Ceará, Fortaleza, Ceará, Brasil. 


\section{KEY-WORDS}

- Stigma.

- Medical Internship.

- Mental Disorder.

- Medical Education.

Recebido em: 4/5/19

Aceito em: 13/5/19

\begin{abstract}
Historically, there is stigma associated to several diseases. There are widespread stigma and prejudice regarding mental illness throughout the world and research has shown that the general population has limited knowledge about mental illness. One mental disorder in particular, schizophrenia, is highly stigmatized. As members of society, medical students are not immune to stigma assigned to people with mental disorders. Several studies have suggested that the psychiatric training, especially when involving direct contact with the patient, such as medical internship, can have a positive impact on the medical student through their direct engagement in patient care, interest in participating in other activities such as therapy group management, case management, and gaining an understanding that patients with psychiatric conditions can be treated successfully. Most doctors, however, receive little training when interacting with mental illness patients. They usually feel uncomfortable or ineffective in communicating with them, even regarding physical complaints. The objective of this study was to evaluate whether the internship in a psychiatric hospital in Fortaleza reduces the stigma of medical students in relation to mental illness. AQ-9 validated questionnaire was applied to medical students in the initial period of the internship in psychiatry and repeated at the end of the period. From a total sample of 88 students, 37 (42\%) were male and 51 (58\%) were female, and the mean age was 24.68 years. There was a noticeable difference between the first three domains of AQ-9, which showed a significant decrease in pity $(p=0.029)$, danger $(p=0.004)$ and fear $(p<0.001)$. Stigma does seem to reside in the student population analyzed and the psychiatry internship of the two medical schools studied significantly reduced the results in three of the 9 domains evaluated. Of the sociodemographic variables, only gender influenced the result. Female students presented higher scores than male students in relation to the fear domain while male students presented a higher average than their female counterparts in relation to segregation. The importance of the psychiatry internship which goes beyond technical learning is reinforced, since it has the capacity to reduce stigma in relation to psychiatric, and especially schizophrenic, patients.
\end{abstract}


da assistência ${ }^{13}$. Essas visões estereotipadas da esquizofrenia excluem pessoas com este diagnóstico do convívio social e até de oportunidades de trabalho ${ }^{14}$.

Os estudantes de Medicina, por também fazerem parte da sociedade, não ficam imunes ao estigma em relação a pessoas com transtornos mentais. Além disso, como futuros médicos, estudantes de Medicina são pessoas com grande potencial para desestigmatizar as atitudes e pensamentos preconceituosos no contexto da saúde ${ }^{15}$.

A importância do ensino de Psiquiatria, tanto na graduação quanto no internato, para a educação de estudantes de Medicina é amplamente discutida e reconhecida em todo o mundo ${ }^{16}$. O treinamento prático em Psiquiatria é uma parte necessária da educação médica geral, sendo que qualquer médico deve ser capaz de diagnosticar, tratar, administrar e encaminhar pacientes com transtornos psiquiátricos ${ }^{17}$. Lyons ${ }^{18}$, numa revisão sistemática sobre o impacto do internato em Psiquiatria, avaliou 26 estudos; o internato dura, em média, 5,5 semanas, com moda de quatro semanas de duração.

Ao se iniciar o estágio prático em Psiquiatria, quando os estudantes entram em contato direto com os pacientes, algumas visões negativas podem ser reforçadas, tais como o risco de violência, o uso de drogas ilícitas e uso excessivo de álcool ${ }^{19-22}$. Vários são os motivos para essas atitudes negativas: falta de informações precisas sobre doenças mentais, falta de contato com indivíduos com doença mental e falta de familiaridade ${ }^{23}$. Em um estudo australiano que aplicou um questionário em seis universidades, os estudantes referiram os pacientes como perigosos e perturbadores ${ }^{24}$. Curtis-Barton e Eagles ${ }^{25}$ constataram em sua pesquisa que a percepção de pior prognóstico dos pacientes psiquiátricos era o fator que mais desencorajava a escolha pela psiquiatria como carreira. Já Cutler et al. ${ }^{26}$ obtiveram o relato dos estudantes de que a interação com os pacientes psiquiátricos é muito estressante.

Elencou-se a interação de alguns fatores para tentar explicar as atitudes dos estudantes, entre eles o ambiente acadêmiCo, as circunstâncias socioculturais, as experiência individuais e os métodos de ensino (internato) ${ }^{27}$. Vários outros estudos, no entanto, sugeriram que a educação psiquiátrica do estudante de Medicina, principalmente as experiências que envolvem contato direto com o paciente, como o internato, pode ter um impacto positivo, como no engajamento direto ao atendimento do paciente, interesse em participar de outras atividades, como terapia grupal e gerenciamento de casos, além do entendimento de que pacientes com condições psiquiátricas podem ser tratados com sucesso ${ }^{15,28,29-31}$. Esse impacto pode ocorrer mesmo nos estudantes que já demonstravam atitudes positivas em relação a doentes mentais ${ }^{32}$.
Constatou-se ainda ressignificação em algumas atitudes dos alunos comparando-se a graduação e o início desta. No entanto, observou-se que a interação dos alunos com pessoas com problemas mentais durante toda a sua educação médica é fundamental para essa melhora e não apenas no internato ${ }^{22}$.

Quanto ao internato, um estudo que avaliou, além do estigma, as atitudes em relação à psiquiatria e a consideração desta como carreira apontou que o impacto apresentado foi modesto. $\mathrm{O}$ estudo também conclui que estratégias para superar o estigma no ensino pré-clínico podem proporcionar habilidades para melhor preparar os alunos para o internato ${ }^{28}$.

A maioria dos médicos, no entanto, recebe pouco treinamento ao interagir com pacientes com doenças mentais. Geralmente, sentem-se desconfortáveis ou ineficazes ao se comunicarem com eles, mesmo sobre queixas físicas ${ }^{33}$. O objetivo deste estudo é avaliar se o estágio do internato em um hospital psiquiátrico de Fortaleza pode diminuir o estigma dos alunos de Medicina em relação à doença mental.

\section{MÉTODO}

Foi aplicado o questionário validado AQ-9 aos estudantes de Medicina no período inicial do estágio do internato em Psiquiatria e repetida a mesma avaliação no final do período. $\mathrm{O}$ internato é "um estágio obrigatório do curso de medicina durante o qual os alunos recebem um treinamento contínuo e intensivo. Geralmente é realizado em instituições de ensino que podem ser vinculadas ou não à faculdade de origem do aluno e sempre há uma supervisão docente que acompanha e avalia o aluno."34.

O estudo foi realizado no Hospital de Saúde Mental Prof. Frota Pinto, onde estudantes de Medicina de duas faculdades estagiam obrigatoriamente no internato de Fortaleza (CE), sendo referência estadual em Psiquiatria. Em média, 20 alunos estagiam a cada mês. Esporadicamente, estudantes de outras universidades também solicitam fazer seus estágios eletivos nesse serviço. A duração do internato é de um mês, incluindo atividades didáticas como sessão clínica e apresentação de casos clínicos e atendimento clínico em emergência, ambulatório e enfermaria. Cada interno acompanha de dois a três pacientes na enfermaria, faz atendimentos ambulatoriais supervisionados em serviços especializados e cumpre uma carga horária de dois a três plantões noturnos na emergência durante o mês do estágio.

As duas avaliações ocorreram no primeiro e no último dia do internato. Os alunos foram convidados a participar da pesquisa respondendo a um questionário sobre estigma, AQ-9, desenvolvido por Corrigan et al. em 2003 (Anexo 1). ${ }^{35}$ Esses autores desenvolveram inicialmente um instrumento de 27 itens, o Questionário de Atribuição (AQ-27), para medir 
os domínios de culpa, raiva, piedade, ajuda, periculosidade, medo, evasão, segregação e coerção. A formulação do questionário foi baseada em dois trabalhos: a Teoria da Atribuição de Causalidade de Bernard Weiner (1995) e o trabalho de Reisenzein (1986). O primeiro autor postula que, quando o indivíduo percebe que a pessoa com necessidade de ajuda tem controle sobre a situação que a levou à solicitação, a intenção de ajuda seria menor, uma vez que o indivíduo teria reações afetivas de culpabilização da vítima e desidentificação, pois o indivíduo, se quisesse, poderia ter evitado chegar àquela situação ${ }^{36}$. $\mathrm{O}$ segundo autor replicou os achados iniciais do primeiro, corroborando a hipótese da mediação do estado afetivo sobre a intenção de ajudar ${ }^{37}$.

Posteriormente, uma versão resumida de nove itens do AQ-27, o AQ-9, foi desenvolvida com as maiores cargas fatoriais, ou seja, questões que apresentaram maiores correlações entre as variáveis originais e os fatores. Os itens do AQ-9 incluem os mesmos domínios do AQ-27. O questionário AQ-9 relata um caso de um paciente com esquizofrenia e as nove perguntas relacionadas ao caso; 1 . Eu sentiria pena do José; 2. Quão perigoso acha que o José é?; 3. Quão assustado com o José se iria sentir?; 4. Acho que o José é culpado da sua condição presente; 5 . Acho que seria melhor para a comunidade do José se ele fosse internado num hospital psiquiátrico; 6. Quão zangado se sentiria com o José?; 7. Qual a probabilidade de que iria ajudar o José?; 8. Eu iria tentar manter-me afastado do José; 9. Acha que o José devia ser forçado a fazer tratamento médico mesmo contra a sua vontade?

As respostas variam de 1 a 9, sendo que 1 corresponde a "não ou nada", e 9 a "muito ou completamente". O resultado produz um escore representativo de cada um dos fatores, sendo o estigma diretamente proporcional ao maior escore, ou seja, admite-se um resultado superior a 1 (um) como existência de estigma.

Nenhum estudante se negou a participar do estudo, sendo que todos assinaram o Termo de Consentimento Livre e Esclarecido (TCLE). Houve aprovação prévia do Comitê de Ética em Pesquisa do Ipade, segundo as normas da Resolução CNS $\mathrm{n}^{\circ} 466$ de 12 de dezembro de 2012, que regulamenta a pesquisa em seres humanos do Conselho Nacional de Saúde - Ministério da Saúde. As entrevistas foram realizadas de abril a agosto de 2017, totalizando 88 estudantes.

Antes de se aplicar o questionário, foi calculado o número adequado de participantes da pesquisa, sendo que o tamanho da amostra calculado foi de 72 alunos. Para o estudo foram realizadas as seguintes análises:

- comparação das nove variáveis relacionadas ao instrumento AQ-9 entre os dois momentos do estudo: início do estágio do internato (pré-intervenção) e final do estágio do internato (pós-intervenção);

- comparação das nove variáveis do instrumento AQ-9 em relação às seguintes características dos alunos: sexo, idade, estado civil, se têm filhos, se sofrem de doença mental, contato com familiar com doença mental no começo do estágio (pré-intervenção).

Na análise estatística, foi utilizado o teste t para amostras emparelhadas com o objetivo de comparar os nove itens do instrumento entre os dois momentos do estudo. Para a comparação das escalas entre as diferentes variáveis de interesse, foi utilizado o teste $t$ de Student ou teste não paramétrico de Mann-Whitney (MW), quando necessário. A correlação linear entre idade e os nove itens do instrumento foi verificada por meio do coeficiente de correlação de Pearson. As análises estatísticas foram realizadas utilizando-se o software estatístico SPSS (versão 20.0). Para todos os testes foi considerado um nível de significância de 5\%.

\section{RESULTADOS}

Do total de 88 estudantes, observou-se que 37 (42\%) eram do sexo masculino e 51 (58\%) eram do sexo feminino. A média de idade foi de 24,68 anos. Considerando-se que, quanto maior o valor do escore obtido, maior a evidência de que há estigma prévio dos estudantes, observa-se que o estigma está presente na amostra do estudo, variando a intensidade segundo a questão avaliada. As respostas poderiam variar de 1 a 9 .

Vale ressaltar que na literatura não há ainda ponto de corte e estratificação de intensidade de estigma em saúde mental, como, por exemplo baixo, moderado ou alto estigma. O que avaliamos é a variação dos escores, como veremos na Tabela 2.

No primeiro quesito, "Eu sentiria pena do José", obteve-se a média de 5,25. A pergunta seguinte, “Quão perigoso acha que o José é?", obteve a média de 3,86 . Na sequência, a terceira pergunta, "Quão assustado com o José se iria sentir?", obteve média de 4,08. No quarto quesito, "Acho que o José é culpado da sua condição presente", a média obtida foi de 1,16 apenas. Na afirmação número 5, "Acho que seria melhor para a comunidade do José se ele fosse internado num hospital psiquiátri$\mathrm{Co}^{\prime \prime}$, a média obtida foi de 2,53. Quando se avaliou o domínio raiva com a pergunta "Quão zangado se sentiria com o José?", a média obtida foi de apenas 1,49. Já na pergunta "Qual a probabilidade de que iria ajudar o José?, obteve-se média de 7,00. $\mathrm{Na}$ afirmativa "Eu iria tentar manter-me afastado do José", a média encontrada foi de 3,08. Já na última pergunta, “Acha que o José devia ser forçado a fazer tratamento médico mesmo contra a sua vontade?", a média obtida foi de 4,40. 


\begin{tabular}{|c|c|}
\hline $\begin{array}{c}\text { TABELA } 1 \\
\text { Avaliação inicial do estigma dos es } \\
\text { Medicina antes do início do internato }\end{array}$ & $\begin{array}{l}\text { tudantes de } \\
\text { pré-exposição) }\end{array}$ \\
\hline & Média pré-estágio \\
\hline 1. Eu sentiria pena do José. & 5,25 \\
\hline 2. Quão perigoso acha que o José é? & 3,86 \\
\hline 3. Quão assustado com o José se iria sentir? & 4,08 \\
\hline $\begin{array}{l}\text { 4. Acho que o José é culpado da sua condição } \\
\text { presente. }\end{array}$ & 1,16 \\
\hline $\begin{array}{l}\text { 5. Acho que seria melhor para a comunidade } \\
\text { do José se ele fosse internado em um hospital } \\
\text { psiquiátrico. }\end{array}$ & 2,53 \\
\hline 6. Quão zangado se sentiria com o José? & 1,49 \\
\hline $\begin{array}{l}\text { 7. Qual a probabilidade de que iria ajudar o } \\
\text { José? }\end{array}$ & 7,00 \\
\hline 8. Eu iria tentar manter-me afastado do José. & 3,08 \\
\hline $\begin{array}{l}\text { 9. Acha que o José devia ser forçado a fazer } \\
\text { tratamento médico mesmo contra a sua } \\
\text { vontade? }\end{array}$ & 4,40 \\
\hline
\end{tabular}

Pode-se observar que houve diferença entre os três primeiros domínios do AQ-9, que evidenciaram, respectivamente, uma diminuição significativa em piedade $(\mathrm{p}=0,029)$, periculosidade ( $p=0,004)$ e medo $(p<0,001)$. Para estes três itens, os estudantes tiveram maior pontuação antes da realização do estágio $(\mathrm{p}<0,05)$. Conforme visto, "Eu sentiria pena do José" diminuiu de uma média de 5,25 para 4,50; "Quão perigoso acha que o José é" diminuiu de 3,86 para 3,05; e "Quão assustado com o José se iria sentir?" diminuiu de 4,08 para 2,88, em média. Nos demais domínios não houve redução significativa após o estágio em Psiquiatria do internato.

Em relação às correlações sociodemográficas e ao nível prévio de estigma antes da intervenção, houve diferença em relação a duas perguntas e ao gênero do estudante. Em média, alunos do sexo feminino apresentaram maior média do que os alunos do sexo masculino em relação ao item "Quão assustado com o José se iria sentir?" ( $p<0,05)$. Já os alunos do sexo masculino apresentaram maior média do que os alunos do sexo feminino em relação ao item "Seria melhor para a comunidade internar José em um hospital psiquiátrico?" ( $\mathrm{p}<$ 0,05), conforme veremos na discussão.

Não houve diferença entre alunos com diferentes estados civis em relação a nenhum domínio ( $p>0,05)$. No entanto, observa-se que havia apenas cinco alunos casados ou com união estável. Também não houve diferença entre alunos com e sem filho em relação a nenhum item do questionário ( $p$ > 0,05). Vale ressaltar, no entanto, que apenas dois alunos tinham filhos.

\begin{tabular}{|c|c|c|c|c|}
\hline \multicolumn{5}{|c|}{$\begin{array}{l}\text { Comparação dos escores obtidos na escala AQ-9 } \\
\text { antes e após estágio do internato em Psiquiatria }\end{array}$} \\
\hline & & \multicolumn{2}{|c|}{ tempo } & \multirow[t]{2}{*}{ p-valor } \\
\hline & & pré & pós & \\
\hline \multirow{5}{*}{ Eu sentiria pena do José. } & $\mathrm{n}$ & 88 & 88 & \multirow{5}{*}{0,029} \\
\hline & média & 5,25 & 4,50 & \\
\hline & $\begin{array}{l}\text { desvio } \\
\text { padrão }\end{array}$ & 2,23 & 2,36 & \\
\hline & mínimo & 1,00 & 1,00 & \\
\hline & máximo & 9,00 & 9,00 & \\
\hline \multirow{4}{*}{ Quão perigoso acha que o José é? } & $\begin{array}{l}\mathrm{n} \\
\text { média }\end{array}$ & $\begin{array}{c}88 \\
3,86\end{array}$ & $\begin{array}{c}88 \\
3,05\end{array}$ & \multirow{4}{*}{0,004} \\
\hline & & 1,95 & 1,81 & \\
\hline & mínimo & 1,00 & 1,00 & \\
\hline & máximo & 8,00 & 8,00 & \\
\hline \multirow{4}{*}{$\begin{array}{l}\text { Quão assustado com o José se iria } \\
\text { sentir? }\end{array}$} & $\begin{array}{l}\mathrm{n} \\
\text { média }\end{array}$ & $\begin{array}{c}88 \\
4,08\end{array}$ & $\begin{array}{c}88 \\
2,88\end{array}$ & \multirow{4}{*}{$<0,001$} \\
\hline & $\begin{array}{l}\text { desvio } \\
\text { padrão }\end{array}$ & 2,18 & 1,87 & \\
\hline & mínimo & 1,00 & 1,00 & \\
\hline & máximo & 9,00 & 9,00 & \\
\hline \multirow{4}{*}{$\begin{array}{l}\text { Acho que o José é culpado da sua } \\
\text { condição presente. }\end{array}$} & $\begin{array}{l}\mathrm{n} \\
\text { média }\end{array}$ & $\begin{array}{c}88 \\
1,16\end{array}$ & $\begin{array}{c}88 \\
1,18\end{array}$ & \multirow{4}{*}{0,845} \\
\hline & $\begin{array}{l}\text { desvio } \\
\text { padrão }\end{array}$ & 0,60 & 0,92 & \\
\hline & mínimo & 1,00 & 1,00 & \\
\hline & máximo & 5,00 & 9,00 & \\
\hline \multirow{5}{*}{$\begin{array}{l}\text { Acho que seria melhor para } \\
\text { a comunidade do José se ele } \\
\text { fosse internado em um hospital } \\
\text { psiquiátrico. }\end{array}$} & $\begin{array}{l}\mathrm{n} \\
\text { média }\end{array}$ & $\begin{array}{c}88 \\
2,53\end{array}$ & $\begin{array}{c}88 \\
2,42\end{array}$ & \multirow{5}{*}{0,719} \\
\hline & desvio & & & \\
\hline & padrão & 2,12 & 2,09 & \\
\hline & mínimo & 1,00 & 1,00 & \\
\hline & máximo & 9,00 & 9,00 & \\
\hline \multirow{4}{*}{$\begin{array}{l}\text { Quão zangado se sentiria com o } \\
\text { José? }\end{array}$} & $\begin{array}{l}\mathrm{n} \\
\text { média }\end{array}$ & $\begin{array}{c}88 \\
1,49\end{array}$ & $\begin{array}{c}88 \\
1,49\end{array}$ & \multirow{4}{*}{$>0,999$} \\
\hline & $\begin{array}{l}\text { desvio } \\
\text { padrão }\end{array}$ & 0,91 & 1,03 & \\
\hline & mínimo & 1,00 & 1,00 & \\
\hline & máximo & 5,00 & 6,00 & \\
\hline \multirow{4}{*}{$\begin{array}{l}\text { Qual a probabilidade de que iria } \\
\text { ajudar o José? }\end{array}$} & $\begin{array}{l}\mathrm{n} \\
\text { média }\end{array}$ & $\begin{array}{c}88 \\
7,00\end{array}$ & $\begin{array}{c}88 \\
7,44\end{array}$ & \multirow{4}{*}{0,074} \\
\hline & $\begin{array}{l}\text { desvio } \\
\text { padrão }\end{array}$ & 1,60 & 1,71 & \\
\hline & mínimo & 3,00 & 1,00 & \\
\hline & máximo & 9,00 & 9,00 & \\
\hline \multirow{4}{*}{$\begin{array}{l}\text { Eu iria tentar manter-me afastado } \\
\text { do José. }\end{array}$} & $\begin{array}{l}\mathrm{n} \\
\text { média }\end{array}$ & $\begin{array}{c}88 \\
3,08\end{array}$ & $\begin{array}{c}88 \\
2.61\end{array}$ & \multirow{4}{*}{0,121} \\
\hline & desvio & 2,02 & 1,99 & \\
\hline & mínimo & 1,00 & 1,00 & \\
\hline & máximo & 9,00 & 9,00 & \\
\hline \multirow{5}{*}{$\begin{array}{l}\text { Acha que o José devia ser forçado } \\
\text { a fazer tratamento médico } \\
\text { mesmo contra a sua vontade? }\end{array}$} & $\mathrm{n}$ & 88 & 88 & \multirow{5}{*}{0,063} \\
\hline & média & 4,40 & 5,16 & \\
\hline & $\begin{array}{l}\text { desvio } \\
\text { padrão }\end{array}$ & 2,74 & 2,72 & \\
\hline & mínimo & 1,00 & 1,00 & \\
\hline & máximo & 9,00 & 9,00 & \\
\hline
\end{tabular}

Pré = primeiro dia do estágio do internato em Psiquiatria. Pós = último dia do estágio do internato em Psiquiatria. A segunda coluna apresenta os valores de $\mathrm{n}$ (número da amostra), a média, desvio padrão, mínimo (1 a 9) e máximo (1 a 9). $\mathrm{p}=$ probabilidade de significância do teste $\mathrm{t}$ de Student. 


\begin{tabular}{|c|c|c|c|c|}
\hline \multicolumn{5}{|c|}{$\begin{array}{c}\text { TABELA } 3 \\
\text { Correlação entre gênero e diminuição } \\
\text { significativa do estigma }\end{array}$} \\
\hline 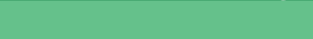 & & \multicolumn{2}{|c|}{ Gênero } & \multirow{2}{*}{ p-valor } \\
\hline & & masculino & feminino & \\
\hline \multirow{5}{*}{ Sentiria pena de José? } & $\mathrm{n}$ & 37 & 51 & \multirow{5}{*}{0,719} \\
\hline & média & 5,35 & 5,18 & \\
\hline & $\begin{array}{l}\text { desvio } \\
\text { padrão }\end{array}$ & 2,23 & 2,26 & \\
\hline & mínimo & 1,00 & 1,00 & \\
\hline & máximo & 9,00 & 9,00 & \\
\hline \multirow{5}{*}{$\begin{array}{l}\text { Quão perigoso acha que } \\
\text { o José é? }\end{array}$} & $\begin{array}{l}\mathrm{n} \\
\text { média }\end{array}$ & $\begin{array}{c}37 \\
3,57\end{array}$ & $\begin{array}{c}51 \\
4,08\end{array}$ & \multirow{5}{*}{0,229} \\
\hline & desvio & & & \\
\hline & padrão & 2,12 & 1,82 & \\
\hline & mínimo & 1,00 & 1,00 & \\
\hline & máximo & 8,00 & 7,00 & \\
\hline \multirow{5}{*}{$\begin{array}{l}\text { Quão assustado com o } \\
\text { José se iria sentir? }\end{array}$} & $\mathrm{n}$ & 37 & 51 & \multirow{5}{*}{$<0,001$} \\
\hline & média & 3,11 & 4,78 & \\
\hline & $\begin{array}{l}\text { desvio } \\
\text { padrão }\end{array}$ & 1,98 & 2,05 & \\
\hline & mínimo & 1,00 & 1,00 & \\
\hline & máximo & 9,00 & 9,00 & \\
\hline \multirow{4}{*}{$\begin{array}{l}\text { Acha que José é } \\
\text { culpado? }\end{array}$} & $\begin{array}{l}\mathrm{n} \\
\text { média }\end{array}$ & $\begin{array}{c}37 \\
1,27\end{array}$ & $\begin{array}{c}51 \\
1,08\end{array}$ & \multirow{4}{*}{0,195} \\
\hline & $\begin{array}{l}\text { desvio } \\
\text { padrão }\end{array}$ & 0,84 & 0,34 & \\
\hline & mínimo & 1,00 & 1,00 & \\
\hline & máximo & 5,00 & 3,00 & \\
\hline \multirow{5}{*}{$\begin{array}{l}\text { Seria melhor para a } \\
\text { comunidade internar } \\
\text { José em um hospital } \\
\text { psiquiátrico? }\end{array}$} & $\mathrm{n}$ & 37 & 51 & \multirow{5}{*}{0,022} \\
\hline & média & 3,19 & 2,06 & \\
\hline & $\begin{array}{l}\text { desvio } \\
\text { padrão }\end{array}$ & 2,60 & 1,55 & \\
\hline & mínimo & 1,00 & 1,00 & \\
\hline & máximo & 9,00 & 6,00 & \\
\hline \multirow{5}{*}{$\begin{array}{l}\text { Quão zangado se } \\
\text { sentiria com o José? }\end{array}$} & $\mathrm{n}$ & 37 & 51 & \multirow{5}{*}{0,245} \\
\hline & média & 1,62 & 1,39 & \\
\hline & $\begin{array}{l}\text { desvio } \\
\text { padrão }\end{array}$ & 1,06 & 0,78 & \\
\hline & mínimo & 1,00 & 1,00 & \\
\hline & máximo & 5,00 & 5,00 & \\
\hline \multirow{4}{*}{$\begin{array}{l}\text { Probabilidade de ajudar } \\
\text { José? }\end{array}$} & $\begin{array}{l}\mathrm{n} \\
\text { média }\end{array}$ & $\begin{array}{c}37 \\
7,27\end{array}$ & $\begin{array}{c}51 \\
6,80\end{array}$ & \multirow{4}{*}{0,178} \\
\hline & $\begin{array}{l}\text { desvio } \\
\text { padrão }\end{array}$ & 1,39 & 1,72 & \\
\hline & mínimo & 4,00 & 3,00 & \\
\hline & máximo & 9,00 & 9,00 & \\
\hline \multirow{5}{*}{ Se afastaria de José? } & $\mathrm{n}$ & 37 & 51 & \multirow{5}{*}{0,167} \\
\hline & média & 2,73 & 3,33 & \\
\hline & $\begin{array}{l}\text { desvio } \\
\text { padrão }\end{array}$ & 1,81 & 2,14 & \\
\hline & mínimo & 1,00 & 1,00 & \\
\hline & máximo & 7,00 & 9,00 & \\
\hline \multirow{5}{*}{$\begin{array}{l}\text { Necessidade de } \\
\text { tratamento médico } \\
\text { contra sua vontade? }\end{array}$} & $\mathrm{n}$ & 37 & 51 & \multirow{5}{*}{0,623} \\
\hline & média & 4,57 & 4,27 & \\
\hline & $\begin{array}{l}\text { desvio } \\
\text { padrão }\end{array}$ & 2,58 & 2,87 & \\
\hline & mínimo & 1,00 & 1,00 & \\
\hline & máximo & 9,00 & 9,00 & \\
\hline
\end{tabular}

A segunda coluna apresenta os valores de $n$ (número da amostra), a média, desvio padrão, mínimo (1 a 9) e máximo (1 a 9). p = probabilidade de significância do teste $t$ de Student.
Também não se constatou nenhuma diferença entre alunos que sofrem ou já sofreram de doença mental nem que tiveram contato com familiar com doença mental em relação a nenhum item. A idade teve correlação muito baixa, próxima a zero, com todos os itens.

\section{DISCUSSÃO}

Avaliar e medir estigma é uma tarefa complexa e existem vários meios e formatos de avaliação distintos e difíceis de serem comparados diretamente. O método de avaliação da estratégia de ensino internato também varia bastante: desde um vídeo sobre a doença mental até estágios com duração de oito semanas. Os questionários utilizados e os dados sociodemográficos avaliados também são variáveis. A maioria dos estudos que tenta medir uma possível diminuição do estigma consegue perceber um resultado significativo, principalmente quando o método a ser avaliado é um estágio no formato de internato $^{30,32,38,39,40}$

Ao se medir o efeito do internato de oito semanas em estudantes de Medicina na China, observou-se melhora significativa na atitude dos alunos em relação ao estigma e à discriminação em relação à psiquiatria e à saúde mental ${ }^{30}$. Uma revisão sistemática de 72 artigos representando 38.364 participantes de 14 países concluiu que tanto a educação como o contato pessoal têm efeitos positivos na redução do estigma da doença mental ${ }^{38}$. Outra intervenção que utilizou apenas duas aulas de três horas com intervalo de uma semana concluiu que os estudantes não consideraram mais os pacientes esquizofrênicos imprevisíveis e perigosos, comparados com sua avaliação pré-intervenção ${ }^{40}$.

No Brasil, poucos estudos tentaram comparar o estigma do estudante de Medicina após o estágio de Psiquiatria no internato. Um estudo aplicou um questionário de 56 itens para avaliar as atitudes dos estudantes de Medicina em relação aos pacientes com doença mental antes e após o estágio em Psiquiatria na cidade do Rio de Janeiro. Concluiu-se que o internato teve efeitos positivos na aceitação social de pessoas com doença mental ${ }^{32}$. Outro estudo que avaliou um internato em urgências em saúde mental para alunos do último ano do curso de Medicina na cidade de Belo Horizonte apresentou diminuição significativa dos fatores segregação, afastamento e medo quando comparado a outras estratégias educacionais ${ }^{39}$. Outro estudo, apesar de não ter avaliado estudantes de Medicina, mas, sim, o público em geral na cidade de São Paulo, constatou que pessoas com esquizofrenia foram percebidas como potencialmente perigosas por $74,2 \%$ dos entrevistados ${ }^{41}$.

Quanto aos domínios especificamente, observou-se que no quesito sobre periculosidade houve diminuição significa- 
tiva de estigma, o que nos faz pensar que o internato cumpriu uma parte importante do seu papel, já que um médico teria muita dificuldade em atender um paciente que achasse perigoso.

A diminuição significativa no domínio medo encontrada em nossa amostra de estudantes retrata o que os preceptores do internato já percebiam subjetivamente ao escutarem o feedback dos alunos. Esse mesmo domínio foi um dos resultados significantes que Pereira ${ }^{39}$ observou em sua tese quando validou o AQ-27 para o português e o testou em alunos de Medicina de Minas Gerais.

A piedade também se destacou como um domínio que sofreu redução significativa do estigma. O contato próximo dos alunos com os pacientes, suas histórias de vida e o relato de seus familiares geralmente comovem os estudantes. Em sua grande maioria, os pacientes do hospital em que foi realizada a pesquisa são de um nível socioeconômico muito baixo, o que gera uma comoção ainda maior quando se soma à própria característica da doença: crônica e às vezes de difícil controle.

Em relação ao domínio medo, alunos do sexo feminino apresentaram maior média do que os alunos do sexo masculino. A pergunta "Quão assustado com o José se iria sentir?" apresentou uma diferença significante em relação ao sexo masculino, apesar de dados de Corrigan et al. ${ }^{38}$ demonstrarem que as mulheres têm menor tendência a estigmatizar do que os homens ${ }^{38}$. Apesar disso, ao se avaliar o domínio bastante estigmatizante como a segregação com a pergunta "Seria melhor para a comunidade internar José em um hospital psiquiátrico?", encontrou-se que os alunos do sexo masculino estigmatizam mais do que os do sexo feminino.

Vinte e cinco por cento dos estudantes referiram que sofrem ou já sofreram com alguma doença mental, o que está de acordo com a literatura brasileira. Os chamados transtornos mentais comuns, que geralmente apresentam sintomas de depressão e ansiedade, podem chegar a uma prevalência de $20 \%$ a $30 \%$ na população geral ${ }^{42}$.

Seria esperado que houvesse maior diminuição do estigma entre os alunos que declararam ter alguma experiência prévia com doenças mentais, como, por exemplo, com algum familiar que sofresse de tais doenças ${ }^{7,43}$. No entanto, nossa amostra não evidenciou essa correlação. Uma possível explicação seria o fato de que o caso apresentado no questionário é de um paciente esquizofrênico, doença que tem prevalência de $1 \%$ da população. Outras doenças, como depressão, ansiedade e TOC, são menos estigmatizadas e possuem prevalência bem superior, chegando a $20 \%-30 \%$. Ou seja, o contato prévio com familiar pode ter sido com um parente deprimido, por exemplo, e o aluno tem que responder sobre um esquizofrê- nico, que tem risco de entrar em crise e apresentar comportamento agressivo.

O estado civil, ter ou não filhos, sofrer de doença mental ou ter tido contato com familiar com doença mental não apresentaram associação com redução de estigma. Outro fator que influencia o estigma seria o nível socioeconômico dos entrevistados. Os estudantes de Medicina avaliados, apesar de não terem sido questionados sobre nível socioeconômico, provavelmente pertencem a classes sociais de nível mais elevado, uma realidade cultural da nossa sociedade, ainda mais que são provenientes de faculdades particulares ${ }^{44}$.

Como limitações do estudo, podemos ressaltar que a nossa amostra é formada apenas por estudantes de Medicina. Alguns estudos também avaliam alunos de Psicologia, Serviço Social e até outras áreas da saúde. Outra limitação consiste no caso apresentado no questionário, pois se refere apenas a um paciente esquizofrênico, não medindo o estigma global da Psiquiatria. Também não foi medido o efeito do estágio no internato de Psiquiatria a longo prazo, ou seja, se as mudanças positivas em relação ao estigma seriam duradouras. Deve-se levar em consideração que, apesar do preenchimento do questionário ter sido de caráter anônimo, uma possível tendência a responder o "moralmente correto" pode ter condicionado algumas respostas. Além disso, os alunos recebem nota ao final do estágio em Psiquiatria, o que também pode ter influenciado, já que é um estágio obrigatório.

\section{CONCLUSÃO}

Admite-se que existe estigma na população de estudantes analisada e que o estágio em Psiquiatria do internato de duas faculdades de Medicina estudadas reduziu significantemente três dos nove domínios avaliados: piedade, periculosidade e medo. Apenas o gênero como dado sociodemográfico influenciou o resultado. Alunos do sexo feminino apresentaram maior média do que os alunos do sexo masculino em relação ao domínio medo, enquanto os alunos do sexo masculino apresentaram maior média do que os alunos do sexo feminino em relação ao domínio segregação. Fortalece-se a importância do estágio em Psiquiatria durante o internato para além do aprendizado técnico, já que tem a capacidade de diminuir o estigma em relação aos pacientes psiquiátricos, principalmente os pacientes esquizofrênicos.

\section{REFERÊNCIAS}

1. Pingani L, Catellani S, Del Vecchio V, Sampogna G, Ellefson SE, Rigatelli M, Fiorillo A, Evans-Lacko S, Corrigan PW. Stigma in the context of schools: analysis of the phenomenon of stigma in a population of university students. BMC Psychiatry. 2016, Feb 9;16:29. 
2. Nunes, ED. Goffman: contribuições para a Sociologia da Saúde. Physis Revista de Saúde Coletiva, Rio de Janeiro, 2009, 19 [ 1 ]: 173-187.

3. Michele L. Pettit. Disease and Stigma: A Review of Literature The Health Educator. Fall 2008, Vol. 40, No.2

4. Wang W, Xiao C, Yao X, Yang Y, Yan, Li S. Psychosocial health and suicidal ideation among people living with HIV/ AIDS: A cross-sectional study in Nanjing, China. PLoS One. 2018 Feb 22;13(2):e0192940. doi: 10.1371/journal. pone.0192940. eCollection.

5. Brewis A,SturtzSreetharan C, Wutich A. Obesity stigma as a globalizing health challenge. Globalization and Health, 2018, 14:20 https:/ / doi.org/10.1186/s12992-018-0337-x.

6. Angermeyer MC, Dietrich S. Public beliefs about and attitudes towards people with mental illness: A review of population studies. Acta Psychiatr. Scand. 2006; 113: 163-197.

7. Economou M, PeppouLE, Louki E , Stefanis CN. Medical students' beliefs and attitudes towards schizophrenia before and after undergraduate psychiatric training in Greece , Psychiatry and Clinical Neurosciences 2012; 66: 17-25.

8. Kato TA,Balhara YPS, Chawla JM, Tateno M, Kanba S. Undergraduate medical students ' attitudes towards psychiatry: An international cross-sectional survey between India and Japan. International Review of Psychiatry, August 2013; 25(4): 378-384.

9. Naeem F, Ayub M, Javed Z, Irfan M, Haral F, Kingdon D. Stigma and psychiatric illness. A survey of attitude of medical students and doctors in Lahore, Pakistan . Journal of Ayub Medical College, Abbottabad ,2006, 18 , 46 - 49.

10. Sartorius N. Stigma and mental health. Lancet, 2007, 370:810-811.

11. Read J, Haslam N, Sayce L, Davies E. Prejudice and schizophrenia: a review of the "mental illness is an illness like any other" approach. Acta PsychiatrScand, 2006, 114:303318.

12. Henry J, von Hippel C, Shapiro L. Stereotype threat contributes to social difficulties in people with schizophrenia. $\mathrm{Br}$ JClin Psychol 2010, 49:31-41.

13. Jones S, Howard L, Thornicroft G. Diagnostic overshadowing': worse physical health care for people with mental illness. Acta PsychiatrScand 2008, 118:169-173.

14. Angermeyer M, Beck M, Dietrich S, Holzinger A. The stigma of mental illness: patients' anticipations and experiences. Int J Soc Psychiatry 2004, 50:153-162.

15. Magliano L, Read J, SagliocchiA, Patalano M, D'ambrosio A, Oliviero N. Differences in views of schizophrenia during medical education: a comparative study of 1st versus 5th-6th year Italian medical students. Soc Psychiatry
Psychiatr Epidemiol. Published on line: 02 November 2012.

16. Kuhn TW, Cohen MJM, Polan HJ, Campbell EC, Clegg KA, Brodkey AC. Standards for Psychiatry Clerkship Directors. Academic Psychiatry,2002, 26:1, Spring.

17. Sher L, New AS. Understanding the Role of psychiatric principles in patient Care: An important Goal of the Medical Student Clerkship in psychiatry. Frontiers in Public Health, February 2016, V 4 ; 30.

18. Lyons Z. Impact of the psychiatry clerkship on medical student attitudes towards psychiatry, and to psychiatry as a career: a systematic review. Acad Psychiatry, 2014; 38:35-42.

19. Dixon RP, Roberts LM, Lawrie S, Jones LA, Humphreys MS. Medical students' attitudes to psychiatric illness in primary care. Med Educ, 2008, 42(11):1080-7.

20. Totic S, Stojiljkovic D, Pavlovic Z, Zaric N, Zarkovic B, Malic L, et al. Stigmatization of 'psychiatric label' by medical and non-medical students. Int J Soc Psychiatry, 2012 Sep;58(5):455-62. doi: 10.1177/0020764011408542. Epub 2011 Jun 30.

21. GiasuddinNA, Levav I, Gal G. Mental health stigma and attitudes to psychiatry among Bangladeshi medical students. Int J Soc Psychiatry published online 9 June 2014.

22. Ay P, Save D, Fidanoglu O. Does stigma concerning mental disorders differ through medical education? A survey among medical students in Istanbul. Soc Psychiatry Psychiatr Epidemiol, 2006, 41:63-67.

23. Corrigan, PW, Green, A, Lundin, R, Kubiak, MA, Penn, DL. Familiarity with and social distance from people who have serious mental illness. Psychiatric Services, 2001, 52, $953-958$

24. Malhi G, Parker G, Parker K, Kirkby K, Boyce P, Yellowlees $\mathrm{P}$, et al. Shrinking away from psychiatry? A survey of Australian medical students' interest in psychiatry. Aust N Z J Psychiatry, 2002;36:416-23.

25. Curtis-Barton M, Eagles J. Factors that discourage medical students from pursuing a career in psychiatry. The Psychiatrist. 2011;35:425-9.

26. Cutler J, Alspector S, Harding K, Wright L, Graham M. Medical students' perceptions of psychiatry as a career choice. Acad Psychiatry. 2006;30(2):144-9.

27. Amini H, Shoar S, TabatabaeeM, Arabzadeh S .The Effect of Clinical Exposure to Patients on Medical Students' Attitude Towards Mental Illness. Iran J Psychiatry Behav Sci. September 2016; 10(3):e1887.

28. Lyons Z, Janca A. Impact of a psychiatry clerkship on stigma, attitudes towards psychiatry, and psychiatry as a career choice. BMC Medical Education, 2015, 15:34. 
29. Wang PW et al. Changes of Explicit and Implicit Stigma in Medical Students during Psychiatric Clerkship. Acad Psychiatry. 2016 Apr;40(2):224-8. doi: 10.1007/s40596-0150432-8. Epub 2015 Oct 8.

30. Shen Y, Dong H, Fan X, Xue ZZZ, GuoLLX, LV H. What can the medical education do for eliminating stigma and discrimination associated with mental illness among future doctors? Effect of clerkship training on chinese students' attitudes. Int'1. J. Psychiatry in Medicine, 2014, Vol. 47(3) 241-254.

31. Lang FU, Kösters M, Lang S, Becker T, Jäger M. Changing Attitude Towards Schizophrenia by Psychiatric Training of Medical Students. PsychiatPrax, 2014; 41: 160-163.

32. Rocha Neto HG, Rosenheck RA, Stefanovics EA, Cavalcanti MT. Attitudes of Brazilian Medical Students Towards Psychiatric Patients and Mental Illness: A Quantitative Study Before and After Completing the Psychiatric Clerkship. Acad Psychiatry. 2017 Jun;41(3):315-319.

33. Jackson JL, Kroenke K. Difficult patients encounters in the ambulatory clinic. Clinical predictors and outcomes. ArchIntMed, 1999; 159: 1069-1075.

34. Lampert JB, Bicudo AM. 10 anos das Diretrizes Curriculares Nacionais dos cursos de graduação em Medicina. Rio de Janeiro: Associação Brasileira de Educação Médica, 2014, p. 57-74.

35. Corrigan P, Markowitz FE, Watson A, Rowan D and Kubiak MA. An Attribution Model of Public Discrimination Towards Persons with Mental Illness. Journal of Health and Social Behavior. 2003; 44 (2); 162-179.

36. KaulfussMA “Atribuição causal para o sucesso e o fracasso em ensinar e eficácia coletiva de professores" Campinas-SP, 2014
37. Pilati R, Leão M, Vieira JN, Fonseca MM. Efeitos da atribuição de causalidade e custo pessoal sobre a intenção de ajuda. Estudos de Psicologia 2008, 13(3), 213-221.

38. Corrigan PW; Morris SB; Michaels PJ; Rafacz JD; Rüsch N. Challenging the public stigma of mental illness: a meta-analysis of outcome studies. Psychiatr Serv. 2012; 63(10):963-73.

39. Pereira AA. Efeito de estratégias educacionais sobre o estigma de alunos de medicina frente ao portador de doença mental. Universidade Federal de Minas Gerais. Faculdade de Medicina Belo Horizonte, 2016.

40. Magliano L, Read J, Sagliocchi A, Oliviero N, D’Ambrosio A, Campitiello F, Zaccaro A, Guizzaro L, Patalano M. “Social dangerousness and incurability in schizophrenia": results of an educational intervention for Medical and Psychology students, Psychiatry Research, http:/ /dx.doi. org/10.1016/j.psychres.2014.06.002

41. Peluso ÉTP, Blay SL. Public stigma and schizophrenia in São Paulo city. RevBras Psiquiatr. 2011;33(2):130-6.

42. Lopes CS et al. ERICA: prevalência de transtornos mentais comuns em adolescentes brasileiros. Rev. Saúde Pública, 2016, vol.50 supl.1 São Paulo Epub Feb 23, 2016

43. Jorm AF, Oh E. Desire for social distance from people with mental disorders: A review. Aust. N. Z. J. Med. 2009; 43: 183-200.

44. Coker EM. Selfhood and social distance: toward a cultural understanding of psychiatric stigma in Egypt. SocSci Med. 2005;61(5): 920-30. 


\section{ANEXO 1 \\ Questionário AQ-9}

Por favor, leia a história do José:

José é um homem com 30 anos de idade, solteiro e com esquizofrenia. Às vezes, ouve vozes e fica perturbado. Ele vive sozinho num apartamento e trabalha como estafeta (estagiário) num grande escritório de advogados. Já foi internado seis vezes devido à sua doença.

Agora, responda a cada uma das seguintes questões sobre o José. Assinale com um X o número que melhor corresponde à sua resposta (numa escala de 1 a 9, em que 1 corresponde a "não ou nada" e 9 corresponde a "muito ou completamente").

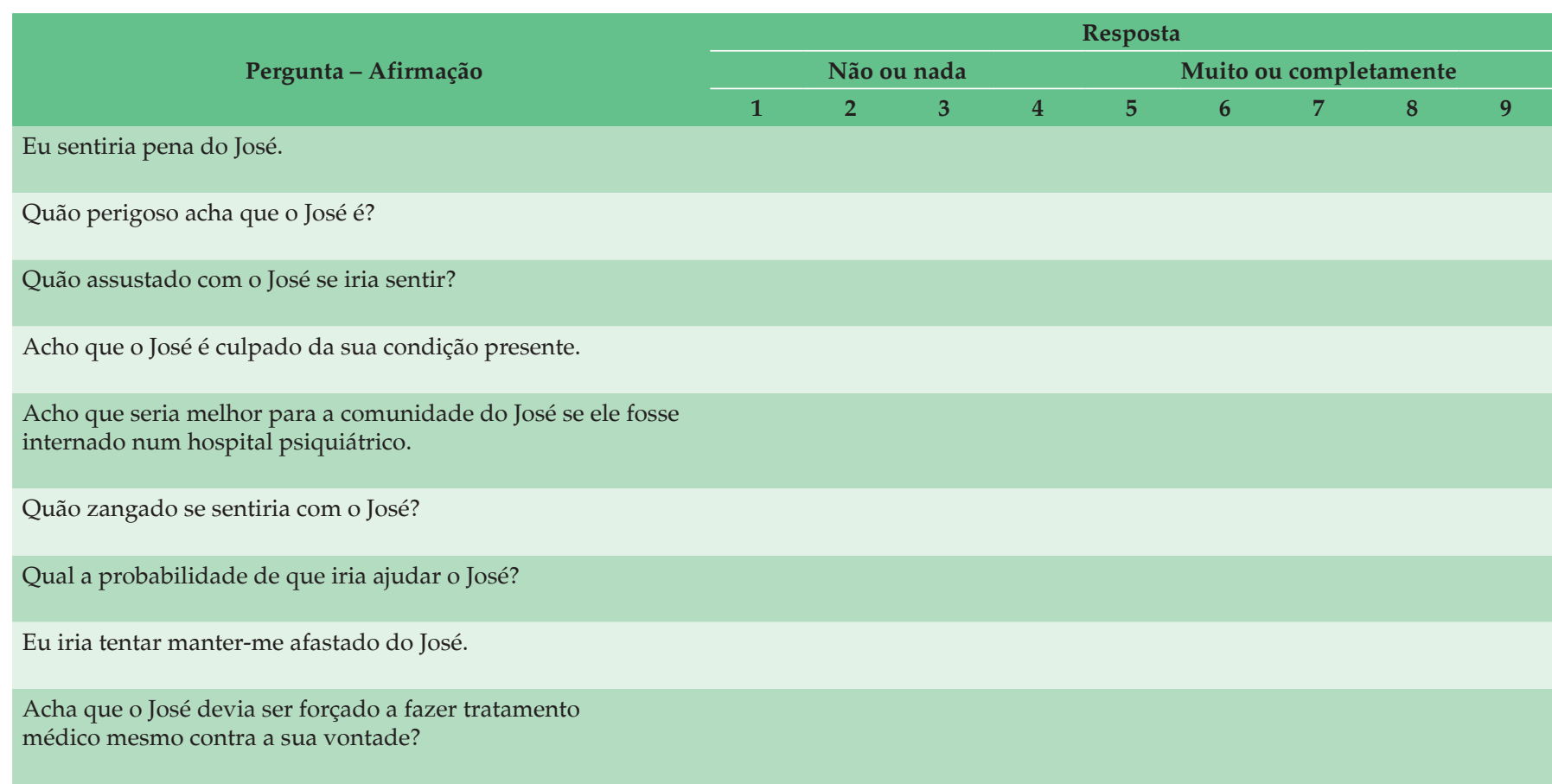

\section{CONTRIBUIÇÃO DOS AUTORES:}

Lorena Pinho Feijó e Sarah Gadelha Motta: revisão de literatura, aplicação dos questionários e discussão dos resultados. Rui Pimenta Saldanha: aplicação dos questionários e tabulação dos dados. Kristopherson Lustosa Augusto e Marcos Kubrusly: discussão dos resultados e revisão do artigo.

\section{CONFLITO DE INTERESSES}

Não há nenhum conflito de interesse.

\section{ENDEREÇO PARA CORRESPONDÊNCIA}

Lorena Pinho Feijó: Avenida Senador Virgílio Távora, 30 ap 802, Meireles, Fortaleza-Ceará, CEP 60170-250 